\title{
Research on the Function Relation of Air-to-Air Missile Body Structure Reliability Optimization
}

\author{
Yuanyuan Kang ${ }^{1, a^{*}}$, Yan Cao ${ }^{1, b}$, Zhou Fang ${ }^{1, c}$ and Guixiang Liu ${ }^{1, d}$ \\ ${ }^{1}$ Department of Mechanical and Electronic Engineering, Xi'an Technological University, Xi'an, \\ China \\ a1300796637@qq.com, bjantonyz@163.com, c27381788@qq.com, d691417473@qq.com \\ *The corresponding author
}

Keywords: Air-to-Air missile; Body structure; Reliability relationship; Reliability optimization

\begin{abstract}
In this paper, the function relation of air-to-air missile body structure reliability optimization is studied. The relationship between structure quality and structure reliability of air-to-air missile is studied deeply, and mathematical model between the reliability of missile structure quality and structure parameters is established, then the reliability of the air-to-air missile body structure is optimized by taking the parameters of the second class missile and some cabin sections as the original data, which put forward a new research method for the reliability optimization of the air-to-air missile body structure.
\end{abstract}

\section{Introduction}

The structure with the best reliability is a structure with minimum quality or minimum cost in the case for a given structural scheme and structural reliability [1]. It is equivalent to the question that the optimal structure in the sense of reliability is the greatest reliability for a given construction scheme and a given structural quality or cost [2]. More specifically, the structural reliability optimization task may be determined by determining the geometric shape of the body structure section and the required reliability based on the analyzed external forces acting on the structure [3]. It indicates that under the action of external load, the structure has the most reasonable arrangement, the quality or the cost of body structure should be minimal.

\section{Relationship between Quality and Reliability of Each Structure of the Air-to-Air Missile}

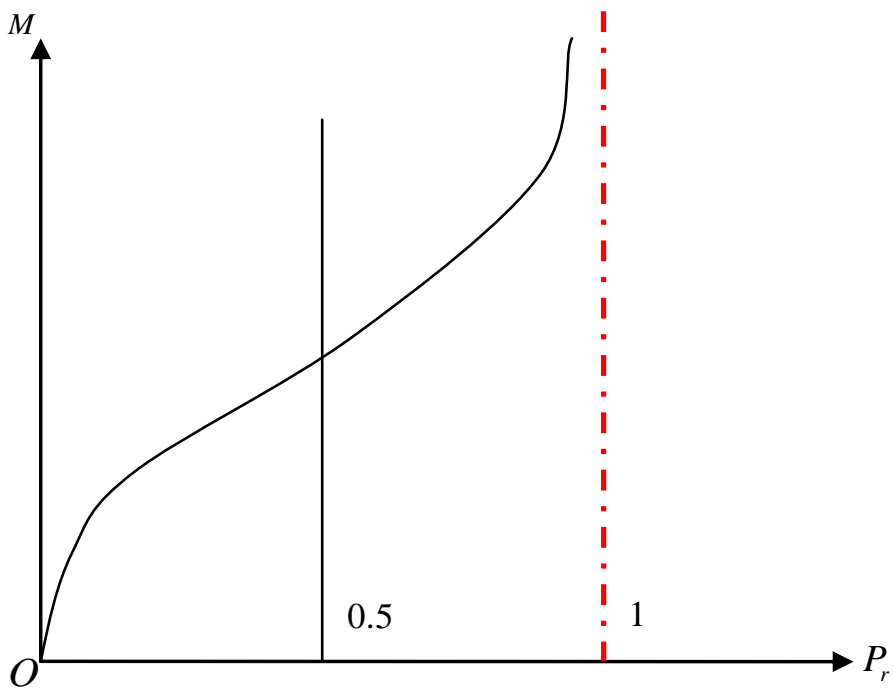

Figure 1. Initial image

In order to determine the relationship between reliability, quality, and cost, the following analysis 
can be performed when the reliability of the body structure is optimized. An increase in structural reliability results in an increase in the mass of the structure [4]. In the case of a cabin structure, the reliability of the structure is equal to 1 when its mass is infinite. With the decrease of cabin structure reliability, cabin structure quality decreases. And the structural mass of the cabin is approximately 0 when the structural reliability of the cabin is equal to zero [5]. It is confirmed by the analysis of the calculation results of a large number of components and cabin structures.

Through the analysis, the relationship between the reliability and the quality of cabin structure is shown in Fig. 1.

As can be seen from Fig. 1, When the reliability $P_{r}$ is greater than 0.5 , the relationship between structural reliability $P_{r}$ and mass is approximately shown as follows.

$$
M=k\left(1-P_{r}\right)^{-\alpha}
$$

During the above formula, $\alpha$ and $k$ are unknown parameters, they shall be derived from two different reliability values of the component or the cabin.

Assuming that the mass of the cabin or the component is $M_{1}$, the corresponding reliability value is $P_{r 1}$, and the corresponding reliability value is $P_{r 2}$ when the mass is $M_{2}$, and then

$$
\begin{aligned}
& M_{1}=k\left(1-P_{r 1}\right)^{-\alpha} \\
& M_{2}=k\left(1-P_{r 2}\right)^{-\alpha}
\end{aligned}
$$

Divide the two sides of the two formulas separately, and the following formula can be get.

$$
\frac{M_{1}}{M_{2}}=\left(\frac{1-P_{r 1}}{1-P_{r 2}}\right)
$$

After taking the natural logarithm both sides of the formula, the following result can be get.

$$
\ln \left(\frac{M_{1}}{M_{2}}\right)=-\alpha \ln \left(\frac{1-P_{r 1}}{1-P_{r 2}}\right)
$$

Therefore $\alpha$ and $k$ are available as follow.

$$
\begin{gathered}
\alpha=\frac{\ln \left(\frac{M_{1}}{M_{2}}\right)}{\ln \left(\frac{1-P_{r 1}}{1-P_{r 2}}\right)} \\
k=\frac{M_{1}}{\left(1-P_{r 1}\right)^{-\alpha}}=\frac{M_{2}}{\left(1-P_{r 2}\right)^{-\alpha}}
\end{gathered}
$$

If the calculation can't be completed in advance, the approximation is desirable.

$$
k=0.67 M_{0}
$$

During the above formula, $M_{0}$ is the structural quality when the safety factor 1.5 is taken to design the structural quality in the traditional design method; and the $\alpha=0.01$.

\section{Reliability Optimization of Air-To-Air Missile Body Structure}

For different parts of the structure, the parameters $\alpha$ and $k$ values are different [6]. Table 1 lists the values of $\alpha$ and $k$ for some sections of the secondary missile and the entire body structure [7]. Eq. 1 is equally applicable to structure at all levels of the missile and body structure. 
In the traditional design method, the relationship between the safety factor and the structural reliability can still be modeled as that between the structure quality and reliability [8], approximate expressions are given.

$$
\gamma_{k}=k_{f}\left(1-P_{r}\right)^{-\alpha}
$$

In the formula, $\gamma_{k}$ is the safety factor used in the traditional design method; $k_{f}$ is the utilization factor.

Based on experience, it can be assumed that the mass of the structure is proportional to the safety factor, that is to say,

$$
M=\left(\frac{M_{0}}{\gamma_{k 0}}\right) \gamma_{k}
$$

Table 1 Parameter values for secondary missile classes and certain cabin sections

\begin{tabular}{cccc}
\hline Structure name & $k / M_{0}$ & $\alpha$ & $k_{f}$ \\
\hline Head & 0.6 & 0.007 & 0.91 \\
Instrument compartment & 0.7 & 0.004 & 1.14 \\
Fuel tank & 0.7 & 0.130 & 1.10 \\
Tail cabin & 0.6 & 0.016 & 0.94 \\
First - level structure & 0.6 & 0.03 & 1.07 \\
Secondary structure & 0.7 & 0.013 & 1.10 \\
Projectile structure & 0.7 & 0.013 & 1.10 \\
\hline
\end{tabular}

In the formula, $M_{0}$ is the structural quality when the safety factor is selected as $\gamma_{k 0}$ in the traditional design method; $\gamma_{k 0}$ is the traditional safety factor used in the first phase of the design, generally take 1.5 . Therefore the parameter $\gamma_{k}$ can be available as follows.

$$
\gamma_{k}=\frac{\gamma_{k 0}}{M_{0}} M=\frac{\gamma_{k 0}}{M_{0}} k\left(1-P_{r}\right)^{-\alpha}=k_{f}\left(1-P_{r}\right)^{-\alpha}
$$

So the parameter $k_{f}$ can be obtained.

$$
k_{f}=k \frac{\gamma_{k 0}}{M_{0}} M=\frac{\gamma_{k 0}}{M_{0}} k\left(1-P_{r}\right)^{-\alpha}=k_{f}\left(1-P_{r}\right)^{-\alpha}
$$

When the structural reliability is the optimal value, the optimal value of the safety factor of the traditional design can be obtained [9].

$$
\gamma_{k}^{*}=k_{f}\left(1-P_{r}^{*}\right)^{-\alpha}
$$

In the formula, the parameter $P_{r}^{*}$ is the optimal value of structural reliability.

It should be noted here that the assumption that the safety factor of the traditional design is proportional to the mass of the structure is not necessarily correct in the case of structural instability [10]. But in general, this assumption gives a good approximation.

If the structural reliability of the body and the safety factor used in the traditional design can't be determined, the following empirical formula can be used to make some estimation in the case of high structural reliability.

$$
-\lg \left(1-P_{r}\right)=25 \gamma_{k}-27
$$

The relationship between structural quality and cost can be expressed as follows: 


\section{$C=\varphi(M)$}

A better and more detailed functional relationship can be given by analyzing the data of the previous design of missile structure. After eliminating quality from the relationship between structural quality and structural reliability and the relationship between structural quality and cost, the relationship between structural quality and structural reliability can be obtained as follows:

$$
C=f\left(P_{r}\right)
$$

This relationship should be used when optimizing the structural reliability according to the cost curve. In particular, there is a relationship between the structural quality and the cost as follows:

$$
C_{u i}=A_{i} M_{i}^{\beta_{i}} n_{i} B_{n_{i}}
$$

In the above formula, $C_{u i}$ is the manufacturing cost of $\mathrm{i}$-th kinds of parts, $A_{i}$ is a constant related to the cost of $\mathrm{i}$-th component, $M_{i}$ is the mass of $\mathrm{i}$-th component; $\beta_{i}$ is the index taking into account the impact of the quality on the cost; $n_{i}$ is the number of $\mathrm{i}$-th component. $B_{n_{i}}$ is the average number of cost reductions for manufacturing $\mathrm{n}$ i-th components, which depends on the production expansion coefficient and the number of manufacturing.

The coefficients $A_{i}, \beta_{i}$ and $B_{n}$ can be determined based on the statistical data. If the number of manufactured components $n_{i}$ is known, the average cost $C_{u i} / n_{i}$ of the component can be determined, and then the cost of the entire body structure can be determined. The cost is generally used in reliability optimization of the body structure.

\section{Conclusion}

In this paper, the mathematical model of function relationship for structural reliability optimization of air-to-air missile body structure is established through in-depth study on the structure of optimal reliability. The reliability of air-to-air missile body structure is optimized based on the established relationship model of structural quality and structural reliability of air-to-air missiles and taking the parameters of secondary missile class and certain cabin sections as the original data, the effect of the optimized reliability in engineering practice is effective. A new research method for the reliability optimization of air-to-air missile body structure is put forward.

\section{Acknowledgments}

The paper is supported by non-traditional machining Key Laboratory Project of Shaanxi Province (15JS041).

\section{References}

[1] W.B. Huang, Y.L. Cai: Optimization of Hull Structure Reliability for a Member of the Cross Section Structure [J]. Journal of Shanghai Jiao Tong University, Vol. 34 (2000) No.1, p.67. (In Chinese)

[2] G.D. Cheng: Optimum Design of Structure Optimization with Reliability [J]. Computational Mechanics, Vol. 50 (1984) No.4, p.109. (In Chinese)

[3] T. Wei: Geometric Structure of Section Stiffness Distribution Optimization Design of a Certain Type of Aircraft (MS., Journal of Northwestern Poly-technical University, China 2006), p.28. (In Chinese)

[4] H. Cao, R. Yuan and Y.Q. Zheng: Research on Structural Reliability Optimization and Its Application Based on Shape Sensitivity [J]. Machinery Manufacturing, Vol. 50 (2012) No.11, p.1. (In Chinese) 
[5] Y. Cao: Study on the Application of the Stochastic Finite Element Method of the Reliability Analysis of Typical Cabin Structure (MS., Harbin Engineering University, China 2012), p.36. (In Chinese)

[6] L. Dong: Optimization Design and Reliability Analysis of Mining Structure Parameters (MS., Central South University, China 2010), p.58. (In Chinese)

[7] H.X. Zhu: Study on the Design of the Typical Missile Cabin Structure Optimization (MS., Second Research Institute of China Aerospace, Second Research Institute of Aerospace Science and Technology Group, China 2015), p.26. (In Chinese)

[8] L. Wang, W.T. Liu: Design of Static Strength Reliability of Aircraft Structure Based on Allowable Safety Factor [J]. Mechanical Strength, Vol. 31 (2009) No.3, p.487. (In Chinese)

[9] Y. Chen: Reliability Optimization Design of Anti-tank Missile Pitch Control System [J]. Journal of Missile and Guidance Journal, Vol. 36 (1995) No.4, p.44. (In Chinese)

[10] J.M. Zhu, M. Cai: Research on Structural Design Method Based on Reliability and Safety Factor [J]. Ship Science and Technology, Vol. 29 (2007) No.3, p.134. (In Chinese) 